\title{
Measuring Charge Transport from Transient Photovoltage Rise Times. A New Tool To Investigate Electron Transport in Nanoparticle Films
}

\author{
Brian C. O’Regan, ${ }^{*}, \dagger$ Klaas Bakker, ${ }^{\ddagger}$ Jessica Kroeze, ${ }^{\dagger}$ Herman Smit, ${ }^{\ddagger}$ Paul Sommeling, ${ }^{\ddagger}$ and \\ James R. Durrant ${ }^{\dagger}$ \\ Department of Chemistry, Imperial College, Exhibition Road, London SW7 2AZ, U.K., and \\ Energy Research Centre Netherlands, Postbus 1, 1755 ZG Petten, The Netherlands
}

Received: May 5, 2006; In Final Form: June 21, 2006

\begin{abstract}
Charge transport rate at open-circuit potential $\left(V_{\text {oc }}\right)$ is proposed as a new characterization method for dyesensitized (DS) and other nanostructured solar cells. At $V_{\text {oc }}$, charge density is flat and measurable, which simplifies quantitative comparison of transport and charge density. Transport measured at $V_{\mathrm{oc}}$ also allows meaningful comparison of charge transport rates between different treatments, temperatures, and types of cells. However, in typical DS cells, charge transport rates at $V_{\mathrm{oc}}$ often cannot be measured by photocurrent transients or modulation techniques due to RC limitations and/or recombination losses. To circumvent this limitation, we show that charge transport at $V_{\mathrm{oc}}$ can be determined directly from the transient photovoltage rise time using a simple, zero-free-parameter model. This method is not sensitive to RC limitation or recombination losses. In trap limited devices, such as DS cells, the comparison of transport rates between different devices or conditions is only valid when the Fermi level in the limiting conductor is at the same distance from the band edge. We show how to perform such comparisons, correcting for conduction band shifts using the density of states (DOS) distribution determined from the same photovoltage transients. Last we show that the relationship between measured transport rate and measured charge density is consistent with the trap limited transport model.
\end{abstract}

\section{Introduction}

The search for inexpensive photovoltaic cells has assumed new urgency as the global climate effects of fossil fuel based energy sources have become hard to ignore. ${ }^{1}$ One way to decrease the cost of solar electricity is to develop cell designs that can use lower purity materials than is presently required for silicon photovoltaic cells. Nanostructured interpenetrating heterojunctions show promise in this direction because they are "majority carrier" devices, where bulk defects and impurities do not serve as recombination centers. Because of this, defects and impurities are tolerated at fairly high levels in majority carrier devices. Interpenetrating heterojunction technologies (a.k.a. "bulk heterojunctions") under investigation include polymer blend cells, dye-sensitized cells of both liquid junction and solid-state varieties, and hybrid organic inorganic composites. For all these cells, a full theoretical description is still under debate. In view of the large number of materials substitutions that might be attempted in these kind of cells, such a theoretical description is sorely needed to aide with further optimization of efficiency and stability. This paper contributes to this discussion with a new characterization method for charge transport in interpenetrating heterojunctions and its application to dye-sensitized cells.

The operating mechanisms of dye-sensitized (DS) cells have been previously described; relevant elements will be summarized below. ${ }^{2,3}$ Light absorption occurs in a monolayer of dye at the

* Corresponding author phone: 0207594 1555; fax: 0207594 5801; e-mail: b.oregan@ic.ac.uk.

Imperial College.

$\doteqdot$ Energy Research Centre Netherlands. interface between a transparent oxide electron conductor (usually $\mathrm{TiO}_{2}$ ) and a transparent electrolyte or hole conductor. Sufficient light absorption is achieved by using a thick layer $(\sim 10 \mu \mathrm{m})$ of nanosized oxide particles $(\sim 20 \mathrm{~nm})$, wherein all the internal surface is coated with the dye. After light absorption, the excited dye molecule injects an electron into the electron conductor, and the thus oxidized dye injects a hole into the electrolyte. After electron and hole creation, efficient operation requires that almost all photogenerated charges are able to escape from the $\mathrm{TiO}_{2}$ /electrolyte layer to their respective contacts, before recombining. This must be true not only at "short circuit" conditions, where it is commonly discussed, but also, more importantly, at voltages as near as possible to $V_{\mathrm{oc}}$. This latter condition is required for a high fill factor, which is required for efficient power conversion.

Transport in dye-sensitized cells has been studied using various techniques but almost always under short circuit conditions (e.g. refs 4-12 and many others). Likewise many measurements of recombination have been made, almost always under open circuit conditions. ${ }^{13-19}$ Since it is well-known that the recombination and transport in these cells are strong functions of the voltage, it is unlikely that a reasonable model of the cell near $V_{\mathrm{oc}}$ can be based on the transport rate measured at short circuit. Thus, the study of transport at and near $V_{\mathrm{oc}}$ is an important contribution to further development of predictive models of cell behavior.

In this paper we show that the charge transport rate at $V_{\mathrm{oc}}$ can be determined from the rise time associated with a small perturbation photovoltage transient taken at open circuit. This new method for measuring transport is important for two reasons. First, photocurrent transients at $V_{\text {oc }}$ are almost always 
RC limited in dye-sensitized electrolyte cells and thus cannot be used to measure transport (vide infra). The rise time of the photovoltage is not limited by the RC discharge constant of the cell and thus circumvents this limitation. Second, in some dye-sensitized cells, the transport rate at $V_{\mathrm{oc}}$ is slower than the recombination rate, thus many charges are lost during photocurrent transients at $V_{\mathrm{oc}}$. The charge transport rate can still be calculated from the difference between the photocurrent and photovoltage decay, but the error can be large if the two are similar. The photovoltage rise occurs on a shorter time scale and is thus less effected by recombination. We show below that if the capacitance of the $\mathrm{TiO}_{2} /$ electrolyte film and the $\mathrm{SnO}_{2} /$ electrolyte contact are both measured, the calculation of the charge transport time from the photovoltage rise time is exact and has no adjustable parameters.

In addition to being useful for modeling purposes, measurement of transport at $V_{\mathrm{oc}}$ allows a much better comparison of transport rates between different cells and conditions (e.g. temperature). ${ }^{20}$ Transport rates in materials with high trap densities are strongly dependent on the ratio of the number of electrons in the traps and in the conduction band. Because of this, quantitative comparisons of transport in DS cells with different treatments, or with materials substitutions, can only be done under conditions where this ratio is the same in both cells. For cells with similar trap state distributions, as used herein, this condition is fulfilled when the position of the Fermi level relative to the conduction band $\left(V_{\mathrm{F}}-V_{\mathrm{cb}}\right)$ is set to the same value in both cells. This is very difficult, if not impossible, to control when measuring transport at short circuit because the Fermi level varies strongly across the film. The gradient of the Fermi level depends inversely on the mobility of the charges, which is the parameter one is attempting to measure. Comparison of transport across different cells is more robust using transport times measured at $V_{\mathrm{oc}}$ because the Fermi level through the $\mathrm{TiO}_{2}$ film is approximately uniform and measurable. By varying the bias light, and thus the $V_{\mathrm{oc}}$, the correct choice of $V_{\mathrm{oc}}$ for each cell can be found that equalizes $V_{\mathrm{F}}-V_{\mathrm{cb}}$ between cells.

\section{Methods}

Dye-sensitized $\mathrm{TiO}_{2}$ cells were made with standard techniques. ${ }^{21}$ Transparent conductive $\mathrm{SnO}_{2}$ glass, LOF Tec 8 or Tec 15 , nominal resistance 8 or $15 \Omega /$ sq, was purchased from Pilkington. $\mathrm{TiO}_{2}$ particles were synthesized from Ti-isopropoxide following the nitric acid/acetic acid route, followed by autoclaving. ${ }^{22,23}$ Layers of the $\mathrm{TiO}_{2}$ particles $(\sim 3 \mu \mathrm{m})$ were deposited by screen printing or doctor blading. For Figures 1, 2 , and 4 the cell geometry was $1 \times 1 \mathrm{~cm}$. The layers were sintered at $450{ }^{\circ} \mathrm{C}$ for $30 \mathrm{~min}$, cooled, placed at $100{ }^{\circ} \mathrm{C}$ into the dye solution, and stored at room temperature for $\sim 16 \mathrm{~h}$. For Figures 3 and 5 the cell geometry was $5 \times 0.5 \mathrm{~cm}$, on Tek 8 glass, which results in series resistance of $\sim 2 \Omega$ and a lower RC time constant. The $\mathrm{TiO}_{2}$ layers were heated by ramping quickly to $570{ }^{\circ} \mathrm{C}$, holding for $10 \mathrm{~min}$, and then allowing to cool slowly. Some films were given an additional treatment in a $50 \mathrm{mM} \mathrm{TiCl}_{4}$ solution for $30 \mathrm{~min}$ at $70{ }^{\circ} \mathrm{C}$, followed by an identical heat treatment. ${ }^{24}$ The dye was applied in a special apparatus as previously described. ${ }^{21}$ The dye was N719 purchased from Solaronix under the name Ruthenium 535. For one cell in Figure 4, a thin solid film of $\mathrm{TiO}_{2}(\sim 50 \mathrm{~nm})$ was deposited on the $\mathrm{SnO}_{2}$ by spray pyrolysis before the porous $\mathrm{TiO}_{2}$ was applied. ${ }^{25}$ For Figures 1, 2, and 4 the electrolyte was R-150 (based on methoxyproprionitrile) purchased from Solaronix. ${ }^{26}$ For Figures 3 and 5 the electrolyte "LI" was acetonitrile, 0.6
$\mathrm{M}$ propylmethylimidazolium iodide, $0.5 \mathrm{M}$ tert-butylpyridine, $0.05 \mathrm{M}$ iodine, and 0.1 M LiI. The electrolyte "GT" substituted $0.1 \mathrm{M}$ guanadinethiocyanate for the LiI.

Photocurrent and photovoltage transients were generally taken using a pump pulse generated by an array of $1 \mathrm{~W}$ red LEDs controlled by a fast solid-state switch, as previously described. ${ }^{19}$ White bias light was supplied by $10 \mathrm{~W}$ "Solarc" lamps (WelchAllyn), which are of the metal halogen type. The bias light was attenuated when needed by neutral density filters. The pulse and bias light were incident on the $\mathrm{SnO}_{2}$ side of the cell, except where mentioned. Pulse lengths of $10-100 \mu$ s were used, with rise and fall times of $\leq 1 \mu \mathrm{s}$. The pulse intensity was controlled in order to keep the height of the photovoltage transient below $10 \mathrm{mV}$. Transients were recorded on a potentiostat (Autolab, Ecochemie) with a resolution of $20 \mu \mathrm{s}$, thus with fitting we could measure transient phenomena with lifetimes $>10 \mu \mathrm{s}$. This system was used due to its superior noise rejection, and no signal averaging was required for transients larger than $1 \mathrm{mV}$. For faster transients, pulses were supplied by either the $660 \mathrm{~nm}$ output of a nitrogen pumped dye laser or the $532 \mathrm{~nm}$ line of a YAG laser. Transients were recorded on an oscilloscope. In all cases, photovoltage decays were single exponential, and the time constant was extracted by fitting. The rise of the photovoltage was occasionally nonexponential at early times but always single exponential for at least the last $50 \%$ of the rise (Figure 1 inset).

Photocurrent transients at $V_{\mathrm{oc}}$ were measured by applying a constant bias light to the cell, while also applying a constant voltage using a voltage source. The voltage source was set to apply exactly the same voltage that the bias light would generate in the open circuit condition. The Fermi level profile inside the cell is thus identical to that at open circuit, and no current flows. A small pulse of additional light was applied as described in the previous paragraph, while the applied voltage was not changed. The pulse causes an increase in the Fermi level inside the $\mathrm{TiO}_{2}$ which then causes a small current to flow through the external circuit. The pulse intensity was set to create a $<2 \mathrm{mV}$ shift in the Fermi level, thus the resulting current transients measure the transport of electrons that occurs at $V_{\text {oc }}$ conditions. The current transients were integrated to charge transients. The last two-thirds of the charge transient were well fit with single exponentials (Figure S1, Supporting Information). For all cells used, the time constants for transport were more than 1 order of magnitude smaller (faster) than those for recombination, thus $<10 \%$ of the electrons were lost to recombination. No adjustment of the transport time constants due to recombination was needed.

It is generally accepted that in dye-sensitized electrolyte cells, at open circuit, the photovoltage (PV) measured at the external contacts is a good measure of the Fermi level position throughout the $\mathrm{TiO}_{2}$ film. The argument is as follows. First, the Fermi level in the iodine/iodide electrolyte is pinned by the large concentration of both species. Further it is generally assumed that recombination occurs homogeneously across the $\mathrm{TiO}_{2} /$ electrolyte film, although there is one interesting dissenting opinion. ${ }^{16}$ This assumption, combined with the effective charge screening by the electrolyte, and the small size of the particles means that, at $V_{\mathrm{oc}}$, the $\mathrm{TiO}_{2}$ Fermi level is at the same distance from the conduction band in all parts of the $\mathrm{TiO}_{2}$. Thus, a small $\Delta V$ created by an additional light pulse measures the same shift of the Fermi level at each point in the $\mathrm{TiO}_{2}$. Because we measure small photovoltage transients, typically $<5 \mathrm{mV}$, the capacitance can be treated as constant during the transient. To calculate the capacitance at each $V_{\mathrm{oc}}$ we have used $C=\Delta Q_{\mathrm{p}} / \Delta V_{\max }$, where 


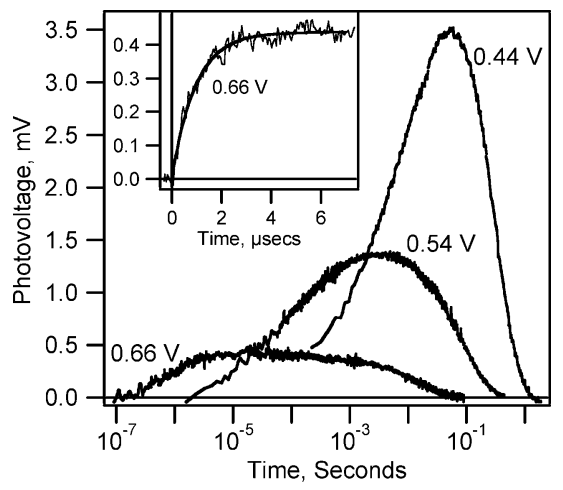

Figure 1. Transient photovoltage vs $\log$ (time) for a dye-sensitized $\mathrm{TiO}_{2}$ cell at varying bias light controlled $V_{\mathrm{oc}}$ 's. Pulse light: $660 \mathrm{~nm}$ dye laser. Pulse intensity same for each transient. White bias light, $0.1-30$ $\mathrm{mW} / \mathrm{cm}^{2}$. Cell area $1 \mathrm{~cm}^{2}$. Inset: transient photovoltage rise vs linear time. Thick line is a single-exponential fit.

$\Delta Q_{\mathrm{p}}$ is the number of electrons injected by the pulse, and $\Delta V_{\max }$ is the peak height of the transient photovoltage. We find $\Delta Q_{\mathrm{p}}$ by integrating the short-circuit photocurrent transient caused by an identical pulse. This will underestimate the actual injected charge by the fraction of electrons that are lost to recombination during transport at short circuit. This error is generally insignificant for DS electrolyte cells but can become important for some solid-state DS cells. ${ }^{27}$ We note that this very simple capacitance measurement agrees well with both impedance spectroscopy and total charge extraction measurements. (Figure S2, Supporting Information, also provides another check.)

In DS cells, the capacitance is a measure of the amount of charge that can be stored in traps in the $\mathrm{TiO}_{2}$. This is because the trap density is large enough, and the Fermi level far enough from the conduction band, that conduction band electrons are a small percentage of the total. The capacitance at a given $V_{\mathrm{oc}}$ is thus proportional to the density of traps states (DOS) at the $\mathrm{TiO}_{2}$ Fermi level corresponding to that $V_{\text {oc }}$. This is true as long as the number of electrons per particle is not large enough to create an electric field in the particle, which holds at all potentials examined in this study. To calculate the DOS (in electron states $/ \mathrm{cm}^{3}$-volt), we have used DOS $=6.24 \times 10^{18} \times$ $C /(d(1-p))$, where $C$ is the capacitance/ $/ \mathrm{cm}^{2}, d$ is the thickness of the $\mathrm{TiO}_{2}$ film in cm, $p$ is the porosity, and the conversion factor is the number of electrons per Coulomb. The total capacitance of the cell also includes a parallel capacitance from the interface between the $\mathrm{SnO}_{2}$ substrate and the electrolyte. This capacitance is known to be $\sim 15 \mu \mathrm{F} / \mathrm{cm}^{2}$. ${ }^{28,29}$

\section{Results and Discussion}

Figure 1 shows typical photovoltage transients for a dyesensitized $\mathrm{TiO}_{2}$ electrolyte cell measured at different open circuit potentials. The data are presented on a log time axis to show both the photovoltage rise and fall behavior over a wide time scale. As the bias light induced $V_{\mathrm{oc}}$ increases, the time constant of the photovoltage decay decreases from 300 to $14 \mathrm{~ms}$. This effect is well-known and corresponds to an increasing recombination rate constant as the Fermi level in the $\mathrm{TiO}_{2}$ moves closer to the conduction band edge. Figure 1 also shows that the photovoltage rise becomes faster as the $V_{\mathrm{oc}}$ increases. Over the voltage range shown, the time constant for the PV rise decreases from $11 \mathrm{~ms}$ to $2.4 \mu \mathrm{s}$.

The observed photovoltage rise will be due to some combination of the displacement of charges in the $\mathrm{TiO}_{2} /$ electrolyte film and the arrival of electrons at the $\mathrm{SnO}_{2}$ contact. Due to the high concentration of mobile ions in the electrolyte, we expect the

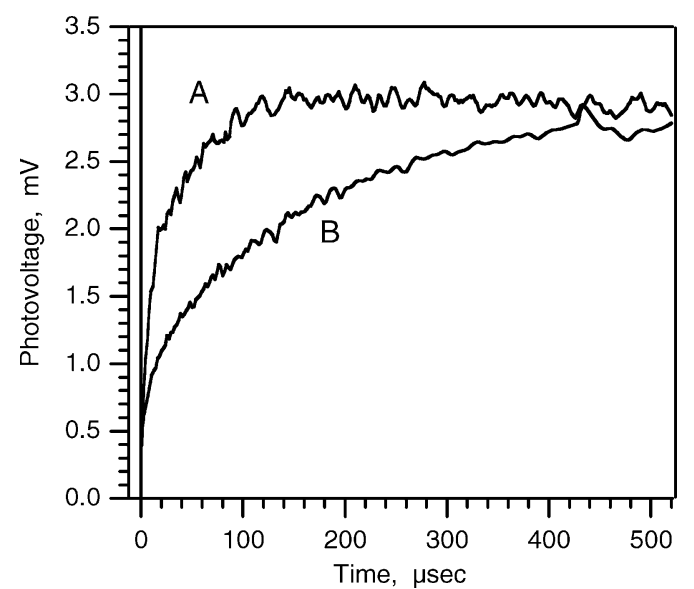

Figure 2. Photovoltage rise times for pulse light incident on the $\mathrm{SnO}_{2}$ side (trace A) and incident on the electrolyte side (B) of a dye-sensitized cell. White bias light incident on the $\mathrm{SnO}_{2}$ side. Pulse light $532 \mathrm{~nm}$ output of YAG laser.

contribution of the displacement effect to be minimal. Figure 2 shows the photovoltage rise measured using a $532 \mathrm{~nm}$ pulse light incident on either the $\mathrm{SnO}_{2}$ or electrolyte side of the cell. Light $(532 \mathrm{~nm})$ is strongly absorbed by the dye, and thus the more charges are created on the side where the light is incident. From Figure 2 it is clear that the photovoltage rise is slower when the charges are created farther from the $\mathrm{SnO}_{2}$ contact. On the other hand, for red light pulses which are absorbed more uniformly across the $\mathrm{TiO}_{2}$ film, the photovoltage rise time is almost independent of pulse illumination direction. These data are consistent with a photovoltage that is established only as the electrons arrive at the $\mathrm{SnO}_{2}$ contact. Further evidence for this is given below.

The exact relation between the photovoltage rise and charge transport can be found by comparing the photovoltage rise times to direct measurements of charge transport at $V_{\text {oc }}$. This is possible using the cells with low RC time constants (see methods) where the transient photocurrent at $V_{\text {oc }}$ can be measured across a wide voltage range. Figure 3 shows the measured transport times for such a cell (filled triangles). The data plotted are the time constants of the integrated photocurrent transients at $V_{\text {oc }}$, as described in the Methods section. Because the measured transport lifetimes are $\geq 10 \times$ shorter than recombination at the same $V_{\text {oc }}$ (circles) the photocurrent transient is a good measure of transport. The transport times decrease with increasing $V_{\mathrm{oc}}$ until the measurement is limited by the RC constant of the cell for $V_{\text {oc }}$ 's above $600 \mathrm{mV}$. Standard cells we have measured are already RC limited for $V_{\text {oc }}$ 's above $450 \mathrm{mV}$ (Figure S2, Supporting Information). Figure 3 also shows the time constants for the photovoltage rise measured on the same cell (filled squares). Comparing the PV rise times to the transport times, we observe that the PV rise is about 1 order of magnitude faster, and the slope with respect to the voltage is slightly steeper. A similar relationship is seen in all cells tested. (Note that the recombination lifetime shows the same trend with voltage as the transport, consistent with transport limited recombination.)

By examination of more than 10 different DS cells, we have found that there is a quantitative relationship between the PV rise time and the transport time. This relationship can be understood by examination of the different circuit elements that give rise to each effect. Figure $3 b$ shows a simplified circuit diagram for a $\mathrm{TiO}_{2} /$ dye/electrolyte cell. We replace the $\mathrm{TiO}_{2} /$ electrolyte interface with a capacitor $C_{\mathrm{TiO}_{2}}$, whose capacitance increases with voltage. Recombination is represented by a 


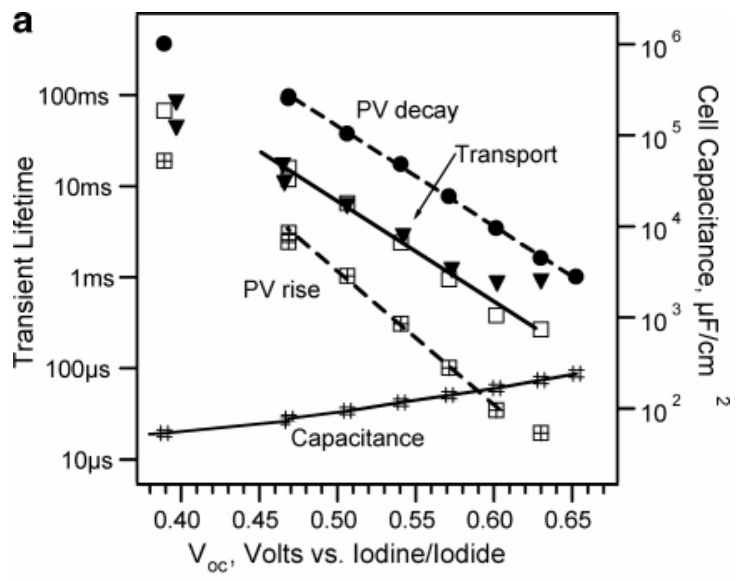

b

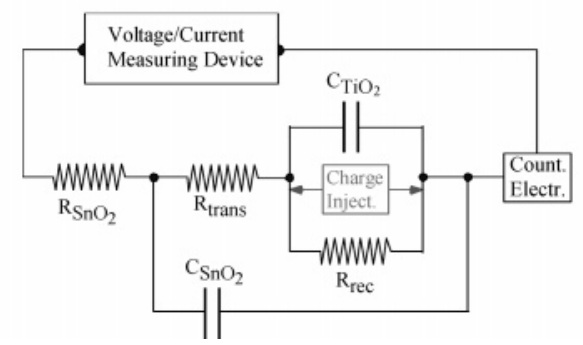

Figure 3. (a) Summary of transient time constants at $V_{\mathrm{oc}}$ for a $\mathrm{TiO}_{2} /$ dye/electrolyte cell: filled triangles are the time constant of the integrated photocurrent transient. Open squares are the transport time calculated from the PV rise time using eq 3 . Also shown is the cell capacitance (\#) determined from the photovoltage peaks (see Methods section). (b) Equivalent circuit for a dye-sensitized $\mathrm{TiO}_{2}$ cell (see text).

voltage dependent resistor, $R_{\text {rec }}$; however, on the time scale of the photovoltage rise virtually no recombination occurs. Transport across the $\mathrm{TiO}_{2}$ we represent as a resistor, $R_{\text {trans }}$, whose resistance decreases with increasing voltage. There is a second capacitance, $C_{\mathrm{SnO}_{2}}$, for the interface between the $\mathrm{SnO}_{2}$ and the electrolyte and a series resistance $R_{\mathrm{SnO}_{2}}$ which combines the resistance of the electrolyte and the lateral resistance of the $\mathrm{SnO}_{2}$ glass. When the cell is at $V_{\text {oc }}$ under bias light, there is a static charge on the capacitors $C_{\mathrm{TiO}_{2}}$ and $C_{\mathrm{SnO}_{2}}$ and no current flowing through the resistors. A pulse of additional light causes some additional electron injection into the $\mathrm{TiO}_{2}$, which is equivalent to adding some extra charge to $C_{\mathrm{TiO}_{2}}$. This extra charge increases the voltage across $C_{\mathrm{TiO}_{2}}$. A certain amount of this charge will move across $R_{\text {trans }}$ to $C_{\mathrm{SnO}_{2}}$ until the voltage drop across both capacitors is the same. Because $C_{\mathrm{TiO}_{2}}$ is much larger than $C_{\mathrm{SnO}_{2}}$, the time constant for this process is that of charging $C_{\mathrm{SnO}_{2}}$. The externally observed voltage measures this charging, which gives a photovoltage rise time expressed by eq 1 .

The photocurrent measurement at $V_{\text {oc }}$ can be described as follows. When the cell is held at a potential equal to $V_{\mathrm{oc}}$, instead of at "open circuit", there is the same static charge on the capacitors, and no current flowing. Again, a pulse of light charges $C_{\mathrm{TiO}_{2}}$, but in this case the excess charge will flow through the external circuit since the applied potential is held at the "prepulse" level. If $R_{\mathrm{SnO}_{2}}$ is smaller than $R_{\text {trans }}$, then the transport across the $\mathrm{TiO}_{2}$ layer is the limiting factor in this discharge. The time constant of the integrated current transient measures this transport, as given in eq 2. If, at a given voltage, $R_{\mathrm{SnO}_{2}}$ is larger than $R_{\mathrm{TiO}_{2}}$, then the cell is termed RC limited, and the charge transient cannot be used to measure transport.

$$
\tau_{\text {rise }}=R_{\text {trans }} \times C_{\mathrm{SnO}_{2}}
$$

$$
\begin{gathered}
\tau_{\text {trans }}=R_{\text {trans }} \times C_{\mathrm{TiO}_{2}} \\
\tau_{\text {trans }}=\tau_{\text {rise }} \times C_{\mathrm{TiO}_{2}} / C_{\mathrm{SnO}_{2}}
\end{gathered}
$$

A slight rearrangement of eqs 1 and 2 gives eq 3 , showing that the transport time through the $\mathrm{TiO}_{2}$ can be easily derived from the photovoltage rise time if $C_{\mathrm{TiO}_{2}}$ and $C_{\mathrm{SnO}_{2}}$ are known. Although this model may seem overly simplified, it is in fact quite accurate. Figure 3a compares the measured times for transport (filled triangles) to the transport times calculated using eq 3 (squares). The agreement is quantitative. We have used $C_{\mathrm{TiO}_{2}}$ measured using the same photovoltage transients (see Methods) and a constant $C_{\mathrm{SnO}_{2}}$ of $15 \mu \mathrm{F}$, taken from the literature. ${ }^{28,29}$ The calculation does not have any adjustable parameters. We have found the same agreement for $\mathrm{TiO}_{2}$ electrolyte cells with several different electrolytes and different $\mathrm{TiO}_{2}$ sources. We note that the relation between the PV rise time and the transport time does not involve any assumptions about the physically correct model for transport.

A further experiment supports the use of eq 3. If the photovoltage rise time is the result of charging of the $\mathrm{SnO}_{2} /$ electrolyte capacitance, then changes in the capacitance of that contact should change the PV rise time. Figure 4 shows the photovoltage transient for two cells, one with and one without a dense $\mathrm{TiO}_{2}$ underlayer on the $\mathrm{SnO}_{2}$. The dense layer is known to reduce the capacitance of the interface $\sim 15$-fold to $\sim 1 \mu \mathrm{F} /$ $\mathrm{cm}^{2}{ }^{28}$ In agreement with eq 3 , we observe that the photovoltage rise is about 20 times faster for the cell containing the dense $\mathrm{TiO}_{2}$ underlayer.

As mentioned in the Introduction, care must be taken when comparing transport rates measured for trap-dominated materials. To remove the effects of differing trap densities, transport times should be compared for a given ratio of trapped to conduction band electrons. Figure 5 illustrates this concept. In Figure 5a the transport time at $V_{\mathrm{oc}}$, derived from PV rise times, is shown for two identical cells containing different electrolytes. One electrolyte (LI) contained 0.1 M LiI, which was replaced in the other electrolyte (GT) by $0.1 \mathrm{M}$ guanadine thiocyanate (full composition, see Methods).

For a given $V_{\text {oc }}$ the cell with the GT electrolyte shows much slower transport (longer extraction times). This might lead one to conclude that the substitution of GT for LI has changed the electron transport properties of the $\mathrm{TiO}_{2}$. However this is a misinterpretation created by using $V_{\mathrm{oc}}$ as the independent variable. Figure $5 b$ shows the measured DOS distributions of the cells shown in Figure 5a. The data show that the DOS curve for the Li electrolyte cell has shifted $100 \mathrm{mV}$ to the left. This

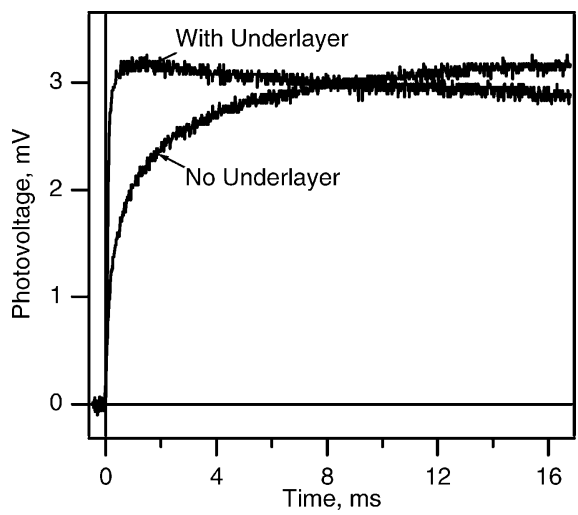

Figure 4. Transient photovoltage rise for a $\mathrm{SnO}_{2} /$ por- $-\mathrm{TiO}_{2} /$ dye/ electrolyte cell, compared to that of a $\mathrm{TiO}_{2} / \mathrm{d}-\mathrm{TiO}_{2} /$ por- $-\mathrm{TiO}_{2} / \mathrm{dye} /$ electrolyte. 

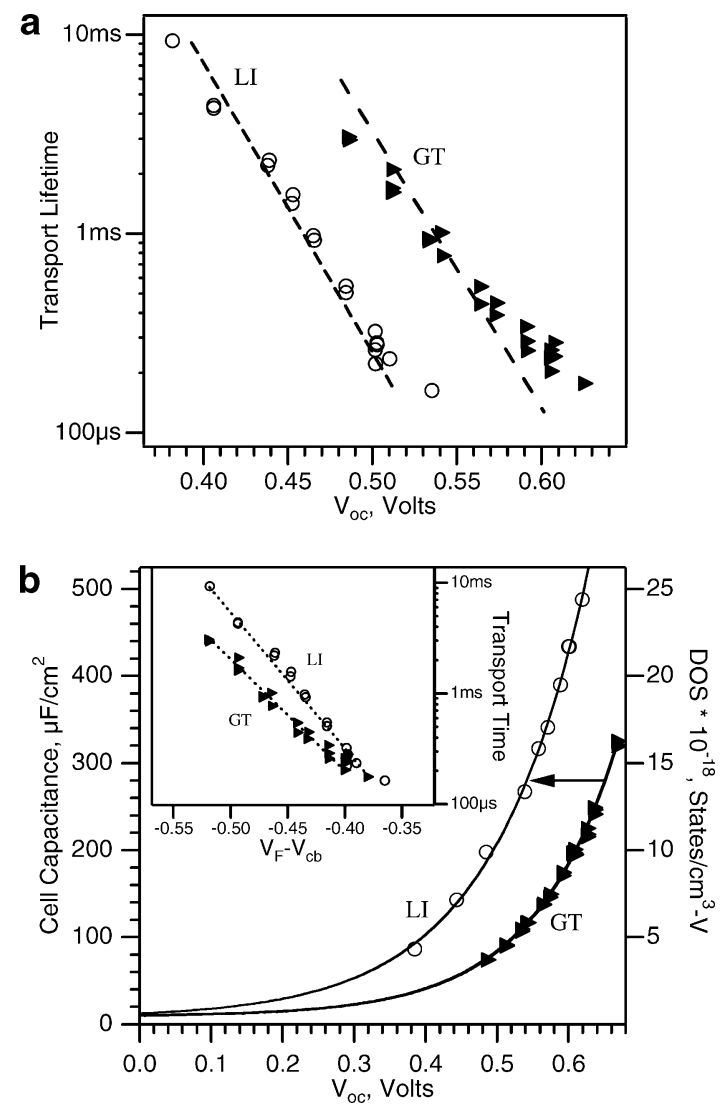

Figure 5. (a) Transport time (charge extraction time) at $V_{\text {oc }}$ for two otherwise identical $\mathrm{TiO}_{2} /$ dye/electrolyte cells with differing electrolytes, LI and GT (for composition see Methods section). Dashed lines have the slope predicted by eq 5 and the DOS data. (b) Measured capacitance (DOS) vs $V_{\text {oc }}$ for the same two cells. Inset: transport times at $V_{\text {oc }}$ plotted vs $\left(V_{\mathrm{F}}-V_{\mathrm{cb}}\right)$ for the same two cells. Dotted lines are only guides.

is almost certainly the result of a $100 \mathrm{mV}$ shift of the $\mathrm{TiO}_{2}$ conduction band downward toward the iodine/iodide potential in the cell containing lithium. The change in the conduction band potential is not surprising as $\mathrm{Li}$ is known to bind to $\mathrm{TiO}_{2}$, increasing the positive charge and thus lowering the band edge. ${ }^{30}$ To correct for this shift, the DOS data can be used to construct a plot of the transport times versus $V_{\mathrm{F}}-V_{\mathrm{cb}}$, the distance between the $\mathrm{TiO}_{2}$ Fermi level and the $\mathrm{TiO}_{2}$ conduction band. Since the DOS distribution curves have the same shape, a given $V_{\mathrm{F}}-V_{\mathrm{cb}}$ gives the same ratio of conduction band to trapped electrons in both cells. To construct the plot, we can assume that the conduction band was $0.9 \mathrm{~V}$ positive of the $\mathrm{I}^{-} / \mathrm{I}_{3}{ }^{-}$ potential in the Li electrolyte and $1.0 \mathrm{~V}$ positive of $\mathrm{I}^{-} / \mathrm{I}_{3}{ }^{-}$in the GS electrolyte. $V_{\mathrm{F}}-V_{\mathrm{cb}}$ can thus be calculated for each $V_{\mathrm{oc}}$ in each cell. The results are shown in Figure $5 \mathrm{~b}$ inset. Figure $5 \mathrm{~b}$ inset implies quite different conclusions than Figure 5a. It is now clear transport in the GT electrolyte cell is actually slightly faster for large $V_{\mathrm{F}}-V_{\mathrm{cb}}$, changing to virtually identical as the Fermi level moves toward the conduction band. Note that the relative position of the transport times in Figure $5 b$ inset is not dependent on the absolute values used for the conduction band potentials, only the difference between them.

It is important to realize that the shift of the conduction band cannot be estimated by the shift of the $V_{\mathrm{oc}}$ at constant light intensity (e.g. 1 sun). For the cells in Figure 5, the one sun $V_{\mathrm{oc}}$ of the GT cell was only $40 \mathrm{mV}$ higher than that of the Li cell. The difference between the shift in the $V_{\mathrm{oc}}$ and the shift in the conduction band is due to changes in the recombination rate constant. In this case, recombination is significantly faster in the GT cell (Figure S3, Supporting Information).
The data in Figure 5 can be used to test transport models which link charge trapping and transport rates. A general model for diffusive transport in defect rich materials such as $\mathrm{TiO}_{2}$ uses an effective diffusion constant which is dependent on the degree of trap filling (eq 4$)^{31}$

$$
\begin{aligned}
D_{\mathrm{eff}}= & \frac{D_{\mathrm{cb}} n_{\mathrm{cb}}(V)}{n_{\mathrm{tot}}(V)} \propto \frac{D_{\mathrm{cb}} e\left(V_{\mathrm{oc}} / k T\right)}{e\left(\beta V_{\mathrm{oc}}\right)} \\
\tau & \propto \frac{d^{2}}{D_{\mathrm{eff}}} \propto e^{(\beta-1 / k T) V_{\mathrm{oc}}}
\end{aligned}
$$

where $D_{\text {eff }}$ is the effective diffusion constant (of all electrons), $D_{\text {cb }}$ is the diffusion constant of electrons in the conduction band, $n_{\mathrm{cb}}$ is the concentration of conduction band electrons, $n_{\mathrm{tot}}$ is the total concentration of electrons, $V\left(=V_{\mathrm{F}}-V_{\mathrm{cb}}\right)$ is the Fermi position level in the $\mathrm{TiO}_{2}, \beta$ describes the curvature of the DOS distribution, $t$ is the transport (electron extraction) time, and $d$ is the thickness of the $\mathrm{TiO}_{2}$ film.

In the last term of eq 4 we have dropped all constants and expressed the DOS distribution as an exponential of voltage with a characteristic curvature $\beta$. This is justified by the exponential fit lines plotted in Figure $5 b$. Under the assumption that charges move only by diffusion, the charge transport time will be related to $D_{\text {eff }}$ via eq 5 . The proportionality constant of $\tau$ to $D_{\text {eff }}$ depends on the experimental details (e.g. light absorption profile) and is not important for the following analysis. Equations 4 and 5 predict that the charge extraction lifetime will be exponentially related to the $V_{\text {oc }}$ and that the slope of $\ln (\tau)$ with respect to $V_{\text {oc }}$ should be $\beta-(1 / k T)$. We have plotted lines with this predicted slope through the transport data on Figure 5a. For the LI cell, the theory fits the data very well, thus further supporting the model. For the GT cell, the transport does not increase as fast as expected with increasing potential. For a few other cells tested, the agreement between the data and eq 5 lies in the range shown in Figure 5a.

\section{Conclusions}

We have shown that electron transport in nanoporous $\mathrm{TiO}_{2}$ can be measured at open circuit by analyzing the rise time of photovoltage transients. We have demonstrated that measured transport times at open circuit are useful for comparing transport in different cells and for verification of proposed transport models. We are aware that the small data set presented here does not resolve the question of the correct model for transport. However, we believe that further measurements of transport and DOS at $V_{\mathrm{oc}}$ are more likely to enable progress in this direction than the current practice of measuring transport at short circuit. There is evidence that recombination between the electron and the dye cation is transport limited in dye-sensitized $\mathrm{TiO}_{2}$ films. It is not yet clear that this paradigm can be extended to recombination between the electron and the electrolyte/hole conductor in all varieties of dye-sensitized cells. Since recombination is virtually always characterized at $V_{\mathrm{oc}}$, comparison of recombination results to transport also measured at $V_{\text {oc }}$ should be a productive approach.

Acknowledgment. Funding for this work was provided by the CEC project "Molycell" (no. 502783).

Supporting Information Available: Integrated photocurrent transients at $V_{\mathrm{oc}}$ showing the single-exponential fits (Figure S1), kinetic and capacitance data for two standard dye-sensitized cells showing the RC limitation on the photocurrent transient at $V_{\mathrm{oc}}$ 
(Figure S2), and recombination lifetimes vs $V_{\mathrm{oc}}$ showing the importance of correcting for the band edge position (Figure S3). This material is available free of charge via the Internet at http:// pubs.acs.org.

\section{References and Notes}

(1) Houghton, J. T.; Ding, Y.; Griggs, D. J.; Noguer, M.; van der Linden, P. J.; Dai, X.; Maskell, K.; Johnson, C. A. Climate Change 2001, The Scientific Basis; Cambridge Unversity Press: Cambridge, U.K., 2001.

(2) Gratzel, M. J. Photochem. Photobiol., C 2003, 4, 145-153.

(3) van Roosmalen, J. A. M.; O'Regan, B. C.; Kroon, J. M.; Sinke, W. C. Handb. Photochem. Photobiol. 2003, 1, 1-48.

(4) O'Regan, B.; Moser, J.; Anderson, M.; Gratzel, M. J. Phys. Chem. 1990, 94, 8720-8726.

(5) Cao, F.; Oskam, G.; Meyer, G. J.; Searson, P. C. J. Phys. Chem. 1996, 100, 17021-17027.

(6) Abayev, I.; Zaban, A.; Fabregat-Santiago, F.; Bisquert, J. Phys. Stat. Solidi A 2003, 196, R4-R6.

(7) Peter, L. M.; Duffy, N. W.; Wang, R. L.; Wijayantha, K. G. U. J. Electronanal. Chem. 2002, 524, 127-136.

(8) Nakade, S.; Matsuda, M.; Kambe, S.; Saito, Y.; Kitamura, T.; Sakata, T.; Wada, Y.; Mori, H.; Yanagida, S. J. Phys. Chem. B 2002, 106 , 10004-10010.

(9) Nakade, S.; Kambe, S.; Kitamura, T.; Wada, Y.; Yanagida, S. J. Phys. Chem. B 2001, 105, 9150-9152.

(10) van de Lagemaat, J.; Frank, A. J. J. Phys. Chem. B 2000, 104, $4292-4294$

(11) Solbrand, A.; Lindstrom, H.; Rensmo, H.; Hagfeldt, A.; Lindquist, S.-E.; Sodergren, S. J. Phys. Chem. B 1997, 101, 2514-2518.

(12) de Jongh, P. E.; Vanmaekelbergh, D. Phys. Rev. Lett. 1996, 77, 3427.

(13) Bailes, M.; Cameron, P. J.; Lobato, K.; Peter, L. M. J. Phys. Chem. B 2005, 109, 15429-15435.

(14) Liu, Y.; Hagfeldt, A.; Xiao, X. R.; Lindquist, S. E. Sol. Energy Mater. Sol. Cells 1998, 55, 267-281.
(15) Kern, R.; Sastrawan, R.; Ferber, J.; Stangl, R.; Luther, J. Electrochim. Acta 2002, 47, 4213-4225.

(16) Zhu, K.; Schiff, E. A.; Park, N. G.; van de Lagemaat, J.; Frank, A. J. Appl. Phys. Lett. 2002, 80(4), 685 .

(17) Green, A. N. M.; Chandler, R. E.; Haque, S. A.; Nelson, J.; Durrant, J. R. J. Phys. Chem. B 2005, 109, 142-150.

(18) Zhang, Z. P.; Zakeeruddin, S. M.; O’Regan, B. C.; Humphry-Baker, R.; Gratzel, M. J. Phys. Chem. B 2005, 109, 21818-21824.

(19) O'Regan, B. C.; Scully, S.; Mayer, A. C.; Palomares, E.; Durrant, J. J. Phys. Chem. B 2005, 109, 4616-4623.

(20) O'Regan, B. C.; Durrant, J. R. J. Phys. Chem. B 2006, 110, 85448547.

(21) Spath, M.; Sommeling, P. M.; van Roosmalen, J. A. M.; Smit, H. J. P.; van der Burg, N. P. G.; Mahieu, D. R.; Bakker, N. J.; Kroon, J. M. Prog. Photovoltaics 2003, 11, 207-220.

(22) O'Regan, B.; Grätzel, M. Nature 1991, 353, 737-740.

(23) Barbé, C. J.; Arendse, F.; Comte, P.; Jirousek, M.; Lenzmann, F.; Shklover, V.; Grätzel, M. J. Am. Ceram. Soc. 1997, 80, 3157-3171.

(24) Nazeeruddin, M. K.; Kay, A.; Rodicio, I.; Humphry-Baker, R.; Muller, E.; Liska, P.; Vlachopoulos, N.; Grätzel, M. J. Am. Chem. Soc. 1993, $115,6382-6390$

(25) Xu, W. W.; Kershaw, R.; Dwight, K.; Wold, A. Mater. Res. Bull. 1990, 25, 1385.

(26) Wang, P.; Klein, C.; Humphry-Baker, R.; Zakeeruddin, S. M.; Gratzel, M. Appl. Phys. Lett. 2005, 86, 123508. 350.

(28) Cameron, P. J.; Peter, L. M. J. Phys. Chem. B 2003, 107, 14394.

(29) Turrion, M.; Bisquert, J.; Salvador, P. J. Phys. Chem. B 2003, 107 , 9397-9403.

(30) Pelet, S.; Moser, J. E.; Grätzel, M. J. Phys. Chem. B 2000, 104, $1791-1795$

(31) Dloczik, L.; Ileperuma, O.; Lauermann, I.; Peter, L. M.; Ponomarev, E. A.; Redmond, G.; Shaw, N. J.; Uhlendorf, I. J. Phys. Chem. B 1997, $101,10281-10289$ 